\title{
Retos y realidades de la participación social en educación básica: Revisión sistemática de bibliografía
}

\author{
Challenges and Realities of Social Participation in Basic Education: A Systematic \\ Literature Review
}

\section{Desafios e realidades da participação social na educação básica: revisão sistemática da literatura}

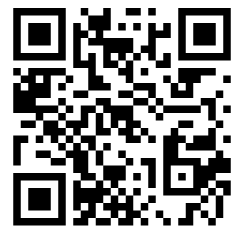

Monserrat Bravo-Delgado

Universidad ICEL

Ciudad de México, México

monserratbravo@icel.edu.mx

http://orcid.org/0000-0001-7969-5930

Leticia Nayeli Ramírez-Ramírez

Universidad Pedagógica Nacional

Ciudad de México, México

nramirez@upn.mx

D http://orcid.org/0000-0002-8113-2368

José Zenón Escobar-Pérez

Universidad del Distrito Federal

Ciudad de México, México

zenon_dgeta@hotmail.com

(D) http://orcid.org/0000-0002-0059-9792

Recibido • Received • Recebido: 17 / 12 / 2018

Corregido • Revised • Revisado: 16 / 05 / 2020

Aceptado • Accepted $\cdot$ Aprovado: 18 / 08 / 2020

\begin{abstract}
Resumen: La política educativa internacional considera la participación social como un referente de análisis y de suma de esfuerzos desde lo local. Su implementación hace posible construir escuelas con una mayor autonomía y vinculación con las necesidades del contexto social, cultural y económico. El presente trabajo tiene por objetivo analizar, de forma sistemática, los estudios recientes realizados en Iberoamérica en relación con la participación social o comunitaria, a fin de conocer las propuestas que se han implementado para promoverla, su estructura como política educativa, sus principales retos y qué prácticas la favorecen o dificultan. Por medio de una búsqueda sistemática en dos bases de datos (SCIELO y Redalyc), se han localizado un total de 759 artículos publicados entre 2013 y 2018. A través de un proceso de filtrado, se consideraron como criterios de inclusión: a) el uso de términos: participación social o participación comunitaria, b) investigaciones referentes al ámbito educativo, c) realizadas en instituciones de nivel básico y d) desarrolladas en Iberoamérica. Se conformó una muestra final de 18 trabajos de investigación, agrupados por categorías: perspectivas de la participación social, descripción de experiencias y evaluación de la participación social. Los principales hallazgos apuntan a una tendencia de implementar la participación social como política
\end{abstract}


http://doi.org/10.15359/ree.24-3.16

http://www.una.ac.cr/educare

educare@una.ac.cr

educativa, que no se ha consolidado, lo cual representa un compromiso teórico que no siempre se concreta en la práctica. Resulta importante, por tanto, generar investigaciones orientadas al establecimiento de propuestas para estimular la creación de espacios y dinámicas participativas.

Palabras claves: Participación social; educación; participación comunitaria; política educativa; educación básica.

\begin{abstract}
The international educational policy considers social participation as a benchmark for the analysis and the addition of efforts at the local level. Its implementation makes it possible to build schools with greater autonomy and connection with the needs of the social, cultural, and economic context. This work aims to systematically analyze recent studies conducted in Ibero-America in relation to social or community participation to know the proposals implemented to promote it, its structure as educational policy, its main challenges, and what practices favor or hinder it. Through a systematic search in two databases (SCIELO and Redalyc), 759 articles published between 2013 and 2018 have been located. Through a filtering process, the following were the criteria to select the articles: a) the use of terms: social participation or community participation; b) research related to the educational field; c) studies conducted in institutions of basic level; and d) studies developed in Ibero-America. Eighteen research works were collected and classified according to the following categories: perspectives of social participation, description of experiences, and evaluation of social participation. The main findings point to a tendency to implement social participation as an educational policy, which has not been consolidated, representing a theoretical commitment that is not always concrete in practice. Therefore, it is important to generate research to establish proposals to stimulate the creation of spaces and participatory dynamics.
\end{abstract}

Keywords: Social participation; education; community participation; educational policy; basic education.

Resumo: A política educacional internacional considera a participação social como um ponto de referência para a análise e os esforços conjuntos a partir do local. Sua implementação faz com que seja possível construir escolas com maior autonomia e articulação com as necessidades do contexto social, cultural e económico. $\mathrm{O}$ objetivo deste trabalho é analisar sistematicamente estudos recentes realizados em Ibero-América, relacionados à participação social ou comunitária, a fim de conhecer as propostas implementadas para promovê-la, sua estrutura como política educacional, seus principais desafios e quais práticas favorecem ou dificultam esta ação. Através de uma pesquisa sistemática de duas bases de dados (SCIELO e Redalyc) foram encontradas um total de 759 itens publicados entre 2013 e 2018. Através de um processo de seleção, foram considerados como critérios de inclusão: a) o uso de termos: envolvimento social e participação comunitária, b) investigação sobre o campo educacional, c) realizadas em instituições de nível básico, d) desenvolvidos em lbero-América. Foram reunidas uma amostra final de 18 trabalhos de pesquisa, agrupados pelas seguintes categorias: perspectivas de participação social, descrição de experiências e avaliação da participação social. As principais conclusões apontam para uma tendência de implementar a participação social como política educacional, que não foi consolidada, o que representa um compromisso teórico que nem sempre é colocado em prática. Torna-se importante, portanto, gerar pesquisa que visa estabelecer propostas para estimular a criação de espaços e dinâmicas participativas.

Palavras-chave: Participação social; educação; participação comunitária; política educacional; educação básica. 
http://doi.org/10.15359/ree.24-3.16

\section{Introducción}

En los albores del siglo XXI, los movimientos sociales, culturales y económicos derivados del fenómeno de globalización han posicionado la participación social en las instituciones educativas como un tema a tratar dentro de las grandes directrices en política educativa a nivel nacional. De esta forma, la escuela ha trascendido su espacio físico al vincularse a la comunidad, a través de diversos mecanismos y formas de participación, lo que le permite fortalecerse como agente socializador e impulsar los alcances de su labor.

En México, el tema de la vinculación entre la escuela y su entorno social no es reciente. Sus antecedentes se remontan a la propuesta de José Vasconcelos como titular de la Secretaría de Educación Pública y Bellas Artes del período 12 de octubre de 1921 al 2 de julio de 1924, quien planteaba la participación de sociedades científicas en materia educativa. Sin embargo, no sería sino hasta 1992 cuando la descentralización educativa, impulsada por el modelo neoliberal y el contexto globalizado establecería formalmente la participación social como política pública en el Acuerdo Nacional para la Modernización de la Educación Básica. A más de 20 años de implementarse, las evaluaciones en participación social en México, realizadas tanto por organismos nacionales como internacionales, la señalan como una problemática a atender dentro del panorama educativo actual.

La Organización para la Cooperación y el Desarrollo Económicos (OCDE) en su informe Education Policy Outlook 2015:Making Reforms Happens, sitúa el porcentaje de toma de decisiones a nivel local y escuela en un $27 \%$ (considerado el valor mínimo en $23 \%$ ). Esto implica que México es uno de los países con bajos niveles de autonomía en materia curricular, evaluación y uso de los recursos. En cambio, las decisiones son tomadas en su mayoría por el gobierno federal y de los Estados (OECD, 2015).

En México, las instancias de participación social en educación básica son los Consejos Escolares de Participación Social, en donde madres y padres, personal docente y directivo, e integrantes de la comunidad cumplen con funciones de consulta, observación y colaboración educativa. A nivel nacional, evaluar sus resultados ha sido responsabilidad por la Secretaría de Educación Pública (SEP) que, de manera periódica, publica un informe sobre el ciclo funcional de los Consejos Escolares de Participación Social en Educación, desarrollado en cuatro fases: conformación, gestión, evaluación y rendición de cuentas. La última valoración señala, para la primera fase, una tasa de cobertura del 86.6 por ciento, incrementado de forma constante en los últimos ciclos escolares. En relación con la cobertura por nivel y servicio educativo, el mayor porcentaje de Consejos Escolares registrados pertenece a preescolar con diferencias mínimas de hasta dos puntos porcentuales en primaria y secundaria. La fase de gestión destaca que el mayor porcentaje de planeación efectiva se realiza en preescolar, con un 30\%, mientras que en primaria y secundaria en promedio alcanzan el $16 \%$ en la realización de esta actividad. En la fase de evaluación, la cobertura total de sesiones y asambleas se sitúa, a nivel nacional, en un 66.1\% para la primera sesión, 61.5 para la segunda sesión y de un 54.1 
http://doi.org/10.15359/ree.24-3.16

http://www.una.ac.cr/educare

educare@una.ac.cr

por ciento para la segunda asamblea. Para la fase de rendición de cuentas, 92,830 de los Consejos Escolares a nivel nacional obtuvieron el informe de transparencia, de un total de 204609 consejos conformados (Secretaría de Educación Pública [SEP], 2016).

Los datos aportados por la SEP dan cuenta de una participación social debilitada, en el territorio nacional, en parte debido a que la autonomía con la cual cuentan es reducida; pero, a su vez, porque pese a haber logrado un alto índice de conformación en los Consejos Escolares, no se han consolidado las actividades previstas para las fases posteriores. Por este motivo, la participación social se ubica como una problemática destacada dentro del panorama educativo actual, por lo cual resulta pertinente llevar a cabo una revisión sistemática de bibliografía, a fin de conocer los aportes de las investigaciones que se han acumulado en los últimos años.

El objetivo de la presente revisión bibliográfica es analizar, de forma sistemática, los estudios recientes (2013-2018) realizados en Iberoamérica en torno a la participación social o comunitaria, a fin de conocer cuáles son las propuestas implementadas para promoverla, de qué manera se estructura la política educativa, cuáles son sus principales retos y qué prácticas la favorecen o dificultan.

\section{Marco teórico}

Al abordar el problema de participación social en la educación, el primer reto que se afronta es su definición, pues el término participar no cuenta con una definición única. Su ambigüedad radica en que no existe claridad en la finalidad de formar parte de algo, en qué proceso, a nombre de qué intereses ni con qué representación. De esta manera, dentro del ámbito educativo, la participación puede implicar aspectos variados e incluso contradictorios (Latapí Sarre, 2005).

Latapí Sarre (2005) plantea que la participación puede asumir cuatro acepciones principales de carácter cualitativo: a) tomar parte en la conducción de la sociedad y en la toma de decisiones que realizan sus gobernantes; b) una condición necesaria para la existencia de la democracia, pues implica desempeñar actividades que la refuerzan, por ejemplo, militar en un partido político u otro tipo de organización; c) en un plano abstracto se considera como un valor objetivo, constitutivo de la convivencia democrática, que va a plasmarse en las instituciones y a inspirar los comportamientos ciudadanos; y d) una actitud subjetiva de la ciudadanía favorable a los procesos democráticos y fundamentada en sus conocimientos y vivencias.

Es importante destacar que el término participación social es empleado, principalmente en el contexto educativo mexicano, desde el planteamiento de su política educativa en sus programas y normatividad, como la participación organizada de la sociedad en la educación. No obstante, en el concepto internacional, el término más empleado es el de participación comunitaria.

El segundo aspecto a esclarecer es el concepto de lo social, ya que enmarca las esferas en donde la participación se desenvuelve y las personas actoras que en él participan. En este sentido, se destaca el trabajo de uno de los autores más representativos de la teoría social, Jurgüen Habermas, quien ha contribuido con sus planteamientos a la comprensión y el desarrollo de la sociedad. 
http://doi.org/10.15359/ree.24-3.16

Habermas plantea, en primera instancia en su teoría, la existencia de dos dimensiones: una sistémica y otra discursiva. Esta última es descrita a través del concepto del mundo de la vida, que constituye el espacio en donde tiene lugar la interacción comunicativa y desde donde genera formas de integración social basadas en la orientación de las acciones. En contraste, la dimensión sistémica da a lugar una estabilización de los contextos de la acción, aquí la integración implica la armonía del conjunto de los efectos causados por las acciones, sin considerar cuáles sean sus intenciones. De esta manera, la sociedad moderna tiene su origen en el proceso histórico-evolutivo de doble diferenciación de ambas esferas. La sociedad moderna se plantea como una formación caracterizada por su pluralidad interna y por la racionalización social, lo que ocasiona que la interacción entre las personas actoras se sujete a la dinámica de dar razones y poder exigirlas (Sierra, 2012). Entonces, la participación social integra, dentro de las formas de participación antes descritas, la interacción de los actores a través de una dinámica comunicativa en donde las acciones están orientadas por sus intenciones, y produciendo una serie de efectos al desarrollarse en conjunto.

La participación de la sociedad en la educación ha tenido, como uno de sus fines, la democratización de los sistemas educativos. Esta es una de las tendencias en la política educativa actual, particularmente dentro de la educación básica, que abarca los niveles de preescolar, primaria y secundaria, tanto públicas como privadas. A través de ella es posible impulsar los procesos de descentralización, al favorecer la autonomía de gestión y la rendición de cuentas ante la comunidad educativa. Las investigaciones recientes, al igual que las organizaciones internacionales, como el Banco Mundial, reconocen la relación existente entre una mayor participación social y la mejora en la calidad de la educación (Perales Mejía y Escobedo Carrillo, 2016).-

\section{Metodología}

El presente trabajo está fundamentado en la metodología de revisión sistemática de bibliografía, definida por Beltrán (2005) como un estudio integrativo, observacional, retrospectivo, en el cual se integran estudios que examinan una misma pregunta, por tanto permite realizar una síntesis racional de la investigación básica a través de estándares rigurosos. Sus pasos son los siguientes:

1. La enunciación de la pregunta a responder.

2. La definición de criterios de inclusión y exclusión.

3. La formulación del plan de búsqueda.

4. El registro de los datos.

5. La evaluación de la calidad de los estudios seleccionados.

6. La interpretación y presentación de los datos. 
http://doi.org/10.15359/ree.24-3.16

http://www.una.ac.cr/educare

educare@una.ac.cr

Esta investigación se desarrolló como una revisión de carácter cualitativo, cuyas características son la presentación de la evidencia en forma descriptiva sin realizar un análisis descriptivo (Aguilera Eguía, 2014). Su diseño se llevó a cabo en tres fases, establecidas a partir de los pasos que caracterizan este tipo de revisión y que se detallan en la Figura 1.

Figura 1: Fases de la revisión sistemática de literatura
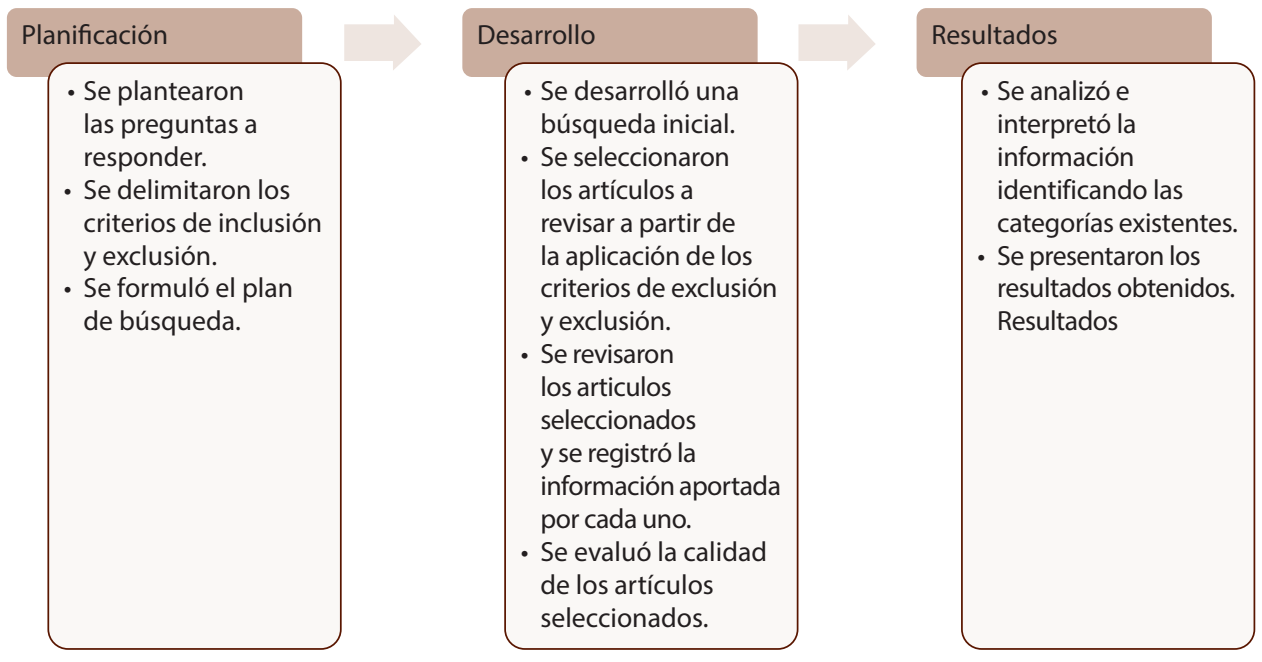

Nota: Elaboración propia.

En una primera fase de planificación, se establecieron como preguntas de investigación las siguientes:

RQ1. ¿Qué tipo de propuestas se han implementado para promover la participación social o comunitaria en educación básica?

RQ2. ¿Qué prácticas o factores favorecen la participación social o comunitaria?

RQ3. ¿Qué prácticas o factores limitan la participación social o comunitaria?

RQ4, ¿Cuáles son los principales retos que afrontan las instituciones educativas en relación a la participación social o comunitaria? 
http://doi.org/10.15359/ree.24-3.16

A partir de los resultados obtenidos, se realizó una selección de artículos con base en los siguientes criterios de inclusión:

a) Artículos que aborden el tema de la participación social o comunitaria.

b) Publicados entre 2013 y 2018.

c) Investigaciones realizadas en Iberoamérica.

d) Abordar la problemática en el ámbito educativo.

e) Estudios desarrollados en el nivel de educación básica.

Como criterios de exclusión se consideraron:

a) Investigaciones que limiten la participación a las prácticas pedagógicas.

b) Revisiones teóricas y ensayos.

Teniendo presentes estos criterios de inclusión y exclusión, se procedió a formular el plan de búsqueda, seleccionando dos bases de datos de acceso abierto (ScIELO y Redalyc), en donde se llevaría a cabo, durante el mes de noviembre de 2018, una pesquisa inicial con las palabras clave participación social y participación comunitaria.

En la fase de desarrollo, a partir de la búsqueda inicial se encontró un total de 759 artículos que cumplían con los criterios de año, país y área temática. Después de revisarse respecto a los criterios de inclusión y exclusión, se seleccionaron 18 artículos para realizar un análisis a profundidad, cuyos resultados se presentan en el siguiente apartado. Así mismo, los artículos seleccionados fueron evaluados en calidad a partir de la revisión del cumplimiento de los criterios de inclusión y exclusión.

\section{Resultados}

En la tercera fase del proceso de revisión, se realizó en primera instancia un análisis contextual en el que se encontró que diez de las investigaciones fueron realizadas en México, tres en Chile, dos en España y una en Costa Rica, Colombia y Argentina respectivamente.

Las palabrasclaveempleadascon mayorfrecuencia fueron:participaciónsocial, participación comunitaria, participación, gestión escolar, democracia, familia, escuela, comunidad.

En relación con el enfoque metodológico se encontró que catorce de los artículos refieren un diseño cualitativo, tres abordaron un diseño cuantitativo y uno abordó un diseño mixto. 


\section{Adicionalmente, se analizaron los hallazgos clasificándolos en tres categorías:}

\section{a) Perspectivas de la participación social}

En esta categoría se incluyen aquellos estudios que dentro de sus hallazgos refieren las creencias, ideas, pensamientos y emociones acerca de la participación social o comunitaria, sus modalidades y mecanismos, así como su relación con la manera en que escuela y comunidad se vinculan (ver Tabla 1).

Tabla 1: Perspectivas de la participación social

\begin{tabular}{|c|c|c|c|c|c|c|}
\hline No. & Autor y año & País & Palabras clave & Objetivo & $\begin{array}{c}\text { Enfoque } \\
\text { metodológico }\end{array}$ & Hallazgos \\
\hline 1 & $\begin{array}{l}\text { Pérez Galván y Ochoa } \\
\text { Cervantes (2017) }\end{array}$ & México & $\begin{array}{l}\text { Aprendizaje, } \\
\text { educación y } \\
\text { democracia, } \\
\text { educación básica. }\end{array}$ & $\begin{array}{l}\text { Indagar las nociones } \\
\text { de participación } \\
\text { en adolescentes y } \\
\text { mostrar la influencia } \\
\text { de un proyecto } \\
\text { en la formación } \\
\text { ciudadana. }\end{array}$ & Cualitativo & $\begin{array}{l}\text { La estructura de las instituciones } \\
\text { limita la participación al determinar } \\
\text { sus formas y espacios. Se requiere } \\
\text { impulsar proyectos participativos } \\
\text { que provean de experiencias } \\
\text { significativas. Se considera necesario } \\
\text { promover la participación del } \\
\text { alumnado en proyectos ciudadanos } \\
\text { que les resulten significativos (Pérez } \\
\text { Galván y Ochoa Cervantes, 2017). }\end{array}$ \\
\hline 2 & $\begin{array}{l}\text { López-Márquez } \\
\text { (2017) }\end{array}$ & México & $\begin{array}{l}\text { Discapacidad, } \\
\text { funcionalidad familiar, } \\
\text { participación escolar }\end{array}$ & $\begin{array}{l}\text { Conocer la } \\
\text { relación entre la } \\
\text { funcionalidad de } \\
\text { las familias que } \\
\text { asisten al CRIT y su } \\
\text { participación escolar. }\end{array}$ & Cuantitativo & $\begin{array}{l}\text { Los padres y madres no desean } \\
\text { integrarse a la sociedad de } \\
\text { padres. Existe mutualismo } \\
\text { entre la capacidad de la escuela } \\
\text { para satisfacer necesidades y la } \\
\text { participación, donde el afecto juega } \\
\text { un papel clave en el apoyo en casa } \\
\text { (López-Márquez, 2017). }\end{array}$ \\
\hline 3 & $\begin{array}{l}\text { Valdés Cuervo y } \\
\text { Sánchez (2016) }\end{array}$ & México & $\begin{array}{l}\text { Familia, participación, } \\
\text { docentes, creencias. }\end{array}$ & $\begin{array}{l}\text { Comprender } \\
\text { las creencias de } \\
\text { docentes que } \\
\text { representan barreras } \\
\text { para la participación } \\
\text { en primarias } \\
\text { públicas. }\end{array}$ & Cualitativo & $\begin{array}{l}\text { El contexto, motivación y creencias se } \\
\text { vinculan a una baja participación. Se } \\
\text { tiene una visión tradicional y limitada } \\
\text { sobre el papel y participación de } \\
\text { la familia, lo que se reduce a la } \\
\text { comunicación familia-escuela, } \\
\text { asistencia a juntas y apoyo en tareas } \\
\text { o dificultades del aprendizaje (Valdés } \\
\text { Cuervo y Sánchez, 2016) }\end{array}$ \\
\hline 4 & $\begin{array}{l}\text { Estrada Ruiz y } \\
\text { Sánchez Parra (2016) }\end{array}$ & México & $\begin{array}{l}\text { Participación social; } \\
\text { consejos escolares; } \\
\text { consejos municipales. }\end{array}$ & $\begin{array}{l}\text { Conocer la cómo } \\
\text { se comprende y } \\
\text { aplica la política } \\
\text { de participación } \\
\text { social, analizando } \\
\text { los sentidos y } \\
\text { representaciones de la } \\
\text { comunidad educativa. }\end{array}$ & Cuantitativo & $\begin{array}{l}\text { La colaboración, aprendizaje y } \\
\text { enseñanza, así como el apoyo formal } \\
\text { constituyen los núcleos centrales } \\
\text { en las representaciones. Se percibe } \\
\text { una participación instrumentalizada } \\
\text { y reducida a la organización e } \\
\text { infraestructura (Estrada Ruiz y } \\
\text { Sánchez Parra, 2016). }\end{array}$ \\
\hline
\end{tabular}

Nota: Elaboración propia. 
http://doi.org/10.15359/ree.24-3.16

http://www.una.ac.cr/educare educare@una.ac.cr

\section{b) Descripción de experiencias de participación social}

Esta categoría está integrada por aquellas investigaciones que describen experiencias específicas de participación social, identifica las formas y mecanismos de colaboración que se implementaron, las relaciones entre las personas actoras participantes (docentes, estudiantes, padres y madres de familia y personal directivo), al igual que sus aciertos, limitantes y los factores que influyeron en los resultados obtenidos (Ver Tabla 2).

Tabla 2: Descripción de experiencias de participación social

\begin{tabular}{|c|c|c|c|c|c|c|}
\hline No. & Autoría y año & País & Palabras clave & Objetivo & $\begin{array}{c}\text { Enfoque } \\
\text { metodológico }\end{array}$ & Hallazgos \\
\hline 1 & $\begin{array}{l}\text { Perales Mejía y } \\
\text { Escobedo Carrillo } \\
(2016)\end{array}$ & México & $\begin{array}{l}\text { Participación social, } \\
\text { gestión escolar, } \\
\text { Consejo Escolar. }\end{array}$ & $\begin{array}{l}\text { Dar a conocer los } \\
\text { resultados de un } \\
\text { estudio en el que } \\
\text { se reconstruye la } \\
\text { participación social } \\
\text { en el CEPS. }\end{array}$ & Cualitativo & $\begin{array}{l}\text { Se da una respuesta parcial a los } \\
\text { Consejos Escolares, a partir de } \\
\text { la experiencia de la comunidad } \\
\text { escolar y tradiciones, aceptando } \\
\text { la autoridad de la directora y } \\
\text { siguiendo con las tradiciones } \\
\text { y experiencia del centro. No } \\
\text { existe difusión ni apoyos } \\
\text { institucionales (Perales Mejía y } \\
\text { Escobedo Carrillo, 2016). }\end{array}$ \\
\hline 2 & $\begin{array}{l}\text { Heras Monner Sans y } \\
\text { Miano (2017) }\end{array}$ & Argentina & $\begin{array}{l}\text { Autogestión, } \\
\text { instituciones } \\
\text { educativas, } \\
\text { participación } \\
\text { política. }\end{array}$ & $\begin{array}{l}\text { Presentar el análisis } \\
\text { de dos experiencias } \\
\text { educativas } \\
\text { autoorganizadas. }\end{array}$ & Cualitativo & $\begin{array}{l}\text { Se presentan dos experiencias } \\
\text { en donde la participación busca } \\
\text { resolver un problema específico, } \\
\text { resulta en la construcción de } \\
\text { nuevos andamiajes jurídico- } \\
\text { legales (Heras Monner Sans y } \\
\text { Miano (2017). }\end{array}$ \\
\hline 3 & Perales Mejía (2014) & México & $\begin{array}{l}\text { Participación social, } \\
\text { comunidad escolar, } \\
\text { democracia. }\end{array}$ & $\begin{array}{l}\text { Reconstruir } \\
\text { las formas de } \\
\text { participación en la } \\
\text { vida cotidiana de un } \\
\text { jardín de infantes. }\end{array}$ & Cualitativo & $\begin{array}{l}\text { La participación social se } \\
\text { concibe como un compromiso } \\
\text { mayormente teórico, existiendo } \\
\text { pocas acciones concretas. } \\
\text { Se detecta la necesidad de } \\
\text { reconocer las particularidades } \\
\text { de los contextos para motivar la } \\
\text { participación (Perales Mejía, 2014). }\end{array}$ \\
\hline 4 & $\begin{array}{l}\text { Pinto Contreras } \\
\text { (2013) }\end{array}$ & Chile & $\begin{array}{l}\text { Construcción } \\
\text { curricular, currículo } \\
\text { emergente, } \\
\text { biodiversidad. }\end{array}$ & $\begin{array}{l}\text { Presentar un } \\
\text { resumen de la } \\
\text { experiencia de } \\
\text { construcción } \\
\text { curricular desde la } \\
\text { cosmovisión Mapuce } \\
\text { Bafkehce. }\end{array}$ & Cualitativo & $\begin{array}{l}\text { Docente que se asumen como } \\
\text { personas educadoras críticas } \\
\text { son aquellas que pueden ser } \\
\text { innovadoras. Los tiempos } \\
\text { académicos e institucionales } \\
\text { no siempre coinciden con } \\
\text { los tiempos culturales de las } \\
\text { poblaciones emergentes en el } \\
\text { currículo (Pinto Contreras, 2013). }\end{array}$ \\
\hline
\end{tabular}

continúa 
http://doi.org/10.15359/ree.24-3.16

http://www.una.ac.cr/educare

educare@una.ac.cr

\begin{tabular}{|c|c|c|c|c|c|c|}
\hline No. & Autoría y año & País & Palabras clave & Objetivo & $\begin{array}{c}\text { Enfoque } \\
\text { metodológico }\end{array}$ & Hallazgos \\
\hline 5 & $\begin{array}{l}\text { Martín y Morales } \\
(2013)\end{array}$ & España & $\begin{array}{l}\text { Relaciones de } \\
\text { colaboración, } \\
\text { entorno, centros } \\
\text { educativos } \\
\text { de educación } \\
\text { secundaria. }\end{array}$ & $\begin{array}{l}\text { Dar a conocer } \\
\text { las relaciones } \\
\text { de colaboración } \\
\text { entre los centros } \\
\text { de educación } \\
\text { secundaria de } \\
\text { Andalucía y su } \\
\text { entorno. }\end{array}$ & Cuantitativo & $\begin{array}{l}\text { Se destaca la colaboración } \\
\text { con otros centros educativos } \\
\text { e instituciones locales. Las } \\
\text { relaciones con asociaciones } \\
\text { y organizaciones no } \\
\text { gubernamentales son } \\
\text { significativas. Las relaciones con } \\
\text { familias, empresas y sindicatos } \\
\text { no han sido significativas (Martín } \\
\text { y Morales, 2013). }\end{array}$ \\
\hline 6 & $\begin{array}{l}\text { Payà Rico y Tormo } \\
\text { Tormo (2016) }\end{array}$ & España & $\begin{array}{l}\text { Política educativa; } \\
\text { participación de los } \\
\text { padres y madres; } \\
\text { asociación de } \\
\text { padres, madres y } \\
\text { profesorado. }\end{array}$ & $\begin{array}{l}\text { Conocer las } \\
\text { percepciones de las } \\
\text { personas agentes } \\
\text { de la educación } \\
\text { en cuanto a la } \\
\text { participación } \\
\text { educativa. }\end{array}$ & Cualitativo & $\begin{array}{l}\text { Se considera la participación } \\
\text { como un mecanismo de } \\
\text { colaboración. El proceso de } \\
\text { transformación ha impulsado } \\
\text { ambas. Como obstáculos se } \\
\text { perciben los horarios de trabajo, } \\
\text { la percepción de intromisión y } \\
\text { el nivel sociocultural; mientras } \\
\text { que se concibe la voluntad para } \\
\text { la transformación y mejora como } \\
\text { un elemento clave para una } \\
\text { participación más activa (Payà } \\
\text { Rico y Tormo Tormo, 2016). }\end{array}$ \\
\hline 7 & $\begin{array}{l}\text { Sánchez Oñate et al. } \\
\text { (2016) }\end{array}$ & Chile & $\begin{array}{l}\text { Participación de los } \\
\text { sujetos apoderados, } \\
\text { expectativas } \\
\text { educativas, } \\
\text { rendimiento } \\
\text { académico. }\end{array}$ & $\begin{array}{l}\text { Conocer la relación } \\
\text { entre la participación } \\
\text { de los padres y } \\
\text { madres de familia } \\
\text { en la educación, } \\
\text { sus expectativas } \\
\text { y el rendimiento } \\
\text { académico. }\end{array}$ & Mixto & $\begin{array}{l}\text { Se encontró una relación } \\
\text { positiva entre el nivel } \\
\text { socioeconómico, el rendimiento } \\
\text { académico y las expectativas. } \\
\text { Se reconocen como barreras las } \\
\text { competencias parentales, el nivel } \\
\text { económico, el desinterés, la falta } \\
\text { de cohesión y una gestión débil } \\
\text { del centro. Entre las experiencias } \\
\text { de participación destacadas } \\
\text { se encuentran las informativas } \\
\text { (Sánchez Oñate et al., 2016). }\end{array}$ \\
\hline 8 & $\begin{array}{l}\text { Gómez y Bermúdez } \\
\text { Urbina (2015) }\end{array}$ & México & $\begin{array}{l}\text { Educación de padres } \\
\text { y madres, escuela- } \\
\text { padres, escuelas } \\
\text { comunitarias. }\end{array}$ & $\begin{array}{l}\text { Explorar los tipos } \\
\text { de participación de } \\
\text { los padres y madres } \\
\text { de familia indígenas } \\
\text { ch'oles en los } \\
\text { procesos escolares. }\end{array}$ & Cualitativo & $\begin{array}{l}\text { La escuela ha favorecido } \\
\text { prácticas que reconocen y } \\
\text { resignifican la organización } \\
\text { comunitaria, fomentando la } \\
\text { participación en acciones de } \\
\text { nivel normativo, organizativo } \\
\text { y de gestión. Se observa una } \\
\text { menor participación en el apoyo } \\
\text { académico, que se relaciona con } \\
\text { el analfabetismo de las madres } \\
\text { y padres (Gómez y Bermúdez } \\
\text { Urbina, 2015). }\end{array}$ \\
\hline
\end{tabular}

Nota: Elaboración propia. 
http://doi.org/10.15359/ree.24-3.16

http://www.una.ac.cr/educare educare@una.ac.cr

\section{c) Evaluación de la participación social}

\section{Esta categoría estuvo integrada por estudios que, a partir de casos concretos, identificaron variables que obstaculizan o favorecen la participación social o comunitaria (Ver Tabla 3).}

Tabla 3: Evaluación de la participación social

\begin{tabular}{|c|c|c|c|c|c|c|}
\hline No. & Autor y año & País & Palabras clave & Objetivo & $\begin{array}{c}\text { Enfoque } \\
\text { metodológico }\end{array}$ & Hallazgos \\
\hline 1 & $\begin{array}{l}\text { Arzola Franco } \\
\text { (2014) }\end{array}$ & México & & $\begin{array}{l}\text { Identificar los } \\
\text { elementos que } \\
\text { intervienen en la } \\
\text { participación y } \\
\text { colegialidad de } \\
\text { escuelas secundarias } \\
\text { federalizadas. }\end{array}$ & Cualitativo & $\begin{array}{l}\text { La estructura educativa local es una } \\
\text { intermediaria entre la SEP y los centros } \\
\text { escolares. El trabajo colegiado y la } \\
\text { participación aun no forman parte } \\
\text { de la cultura escolar, debido a que la } \\
\text { homogenización de las prácticas y } \\
\text { los mecanismos defensivos limitan su } \\
\text { evolución (Arzola Franco, 2014). }\end{array}$ \\
\hline 2 & $\begin{array}{l}\text { Garcés Meneses } \\
(2017)\end{array}$ & Colombia & $\begin{array}{l}\text { Educación, gobierno } \\
\text { escolar, democracia, } \\
\text { participación. }\end{array}$ & $\begin{array}{l}\text { Identificar los } \\
\text { aspectos legales } \\
\text { que limitan la } \\
\text { participación } \\
\text { estudiantil y su } \\
\text { conocimiento acerca } \\
\text { de la participación } \\
\text { democrática a partir } \\
\text { del gobierno escolar. }\end{array}$ & Cualitativo & $\begin{array}{l}\text { La participación política del alumnado } \\
\text { es una figura decorativa, pues se } \\
\text { decide en forma administrativa. } \\
\text { Es necesario un rol definido para } \\
\text { el alumnado que impulse su } \\
\text { empoderamiento y entendimiento } \\
\text { democrático, que permita superar } \\
\text { las prácticas tradicionalistas (Garcés } \\
\text { Meneses, 2017). }\end{array}$ \\
\hline 3 & Hernández (2013) & México & $\begin{array}{l}\text { Política educativa, } \\
\text { participación social, } \\
\text { educación básica. }\end{array}$ & $\begin{array}{l}\text { Ofrecer un } \\
\text { acercamiento a } \\
\text { la incorporación } \\
\text { de la política de } \\
\text { participación social } \\
\text { en la educación } \\
\text { básica. }\end{array}$ & Cualitativo & $\begin{array}{l}\text { Se presenta una simulación como } \\
\text { respuesta a lo normativo. El } \\
\text { liderazgo se fortalece cuando la } \\
\text { comunidad escolar es tomada en } \\
\text { cuenta e impulsa el reconocimiento } \\
\text { de la importancia de la participación } \\
\text { social. Cuando las comunidades son } \\
\text { informadas, participan de forma } \\
\text { más activa en la toma de decisiones } \\
\text { (Hernández, 2013). }\end{array}$ \\
\hline 4 & $\begin{array}{l}\text { Ramírez-González } \\
\text { (2015) }\end{array}$ & Costa Rica & $\begin{array}{l}\text { Relación escuela- } \\
\text { comunidad, } \\
\text { perfil docente } \\
\text { rural, evaluación } \\
\text { diagnóstica. }\end{array}$ & $\begin{array}{l}\text { Presentar los } \\
\text { resultados de } \\
\text { una evaluación } \\
\text { diagnóstica } \\
\text { participativa, } \\
\text { sobre la relación } \\
\text { entre la escuela } \\
\text { y la comunidad } \\
\text { educativa. }\end{array}$ & Cualitativo & $\begin{array}{l}\text { Se desconoce la existencia de } \\
\text { un proyecto institucional y las } \\
\text { necesidades de la escuela. Las } \\
\text { relaciones entre escuela y comunidad } \\
\text { carecen de liderazgo y organización } \\
\text { en ambas direcciones, existe malestar } \\
\text { y distanciamiento entre ambas } \\
\text { instancias a causa de omisiones en } \\
\text { las directrices y falta de comunicación } \\
\text { (Ramírez-González, 2015). }\end{array}$ \\
\hline
\end{tabular}

continúa 
http://doi.org/10.15359/ree.24-3.16

http://www.una.ac.cr/educare

educare@una.ac.cr

\begin{tabular}{|c|l|l|l|l|l|l|}
\hline No. & \multicolumn{1}{|c|}{ Autor y año } & \multicolumn{1}{|c|}{ País } & \multicolumn{1}{|c|}{ Palabras clave } & \multicolumn{1}{c|}{ Objetivo } & \multicolumn{1}{c|}{$\begin{array}{c}\text { Enfoque } \\
\text { metodológico }\end{array}$} & \\
\hline 5 & $\begin{array}{l}\text { Barrientos Piñeiro } \\
\text { et al. (2016) }\end{array}$ & Chile & $\begin{array}{l}\text { Participación, familias, } \\
\text { comunidad. }\end{array}$ & $\begin{array}{l}\text { Describir, analizar } \\
\text { y comprender el } \\
\text { papel de directores } \\
\text { y directoras de } \\
\text { escuelas básicas en } \\
\text { la promoción de la } \\
\text { participación social. }\end{array}$ & Cualitativo & $\begin{array}{l}\text { Directores y directoras reconocen } \\
\text { que la participación es un } \\
\text { proceso fundamental, si bien su } \\
\text { estilo directivo es mayormente } \\
\text { informativo. Identifican como } \\
\text { dificultades los niveles de } \\
\text { participación, compromiso, la visión } \\
\text { de las familias y una débil relación } \\
\text { de confianza (Barrientos Piñeiro et } \\
\text { al., 2016). }\end{array}$ \\
\hline 6 & $\begin{array}{l}\text { Martin y Guzmán } \\
\text { Flores (2016) }\end{array}$ & México & $\begin{array}{l}\text { Participación social en } \\
\text { educación, escuela y } \\
\text { comunidad, la familia } \\
\text { y la escuela, }\end{array}$ & $\begin{array}{l}\text { Analizar la aparente } \\
\text { pasividad en la } \\
\text { participación social } \\
\text { desde la relación } \\
\text { entre padres, madres } \\
\text { y personal docente. }\end{array}$ & Cualitativo & $\begin{array}{l}\text { La ausencia de medidas para dar } \\
\text { seguimiento a los CEPS los ha } \\
\text { convertido en un experimento } \\
\text { fallido, el equipo de docentes ha } \\
\text { mostrado resistencias y los padres } \\
\text { y madres no se han incorporado } \\
\text { realmente. Se identifica como barrera } \\
\text { las condiciones socioeconómicas } \\
\text { de las zonas más pobres (Martin y } \\
\text { Guzmán Flores, 2016). }\end{array}$ \\
\hline
\end{tabular}

Nota: Elaboración propia.

Respecto a las preguntas de investigación, el análisis arroja lo siguiente:

\section{RQ1. ¿Qué tipo de propuestas se han implementado para promover la participación social o comunitaria en educación básica?}

Si bien en la mayor parte de las investigaciones se analiza la participación social en relación con las estructuras establecidas a través de los sistemas educativos, tres de ellas plantean propuestas alternativas que permiten abordar la participación en diferentes dimensiones. La primera de ellas es en la dimensión curricular, donde se muestra que es posible integrar la cosmovisión de una comunidad indígena chilena en la construcción de un currículo emergente, a partir del diálogo con las personas actoras que conforman la comunidad educativa: docentes, autoridades, miembros de la comunidad con interés en el proyecto. El resultado es un diseño curricular pertinente, relevante, integral y diversificado que, en su proceso de construcción, permitió desarrollar la capacidad de comprensión y reflexión en sus participantes (Pinto Contreras, 2013). La segunda propuesta es la desarrollada en Valencia, que consistió en la aplicación del modelo comunidades de aprendizaje que se basa en un conjunto de actuaciones educativas de éxito orientadas a la transformación social y educativa del centro escolar. Sus resultados fueron positivos al sensibilizar e impulsar la participación de las mujeres y hombres agentes de la educación, abriendo espacios de diálogo y formación para la participación (Payà Rico y Tormo Tormo, 2016). La tercera propuesta es sobre la autogestión de dos centros 
http://doi.org/10.15359/ree.24-3.16

educativos en Argentina, en donde los resultados apuntan a la generación de una configuración territorial propia, al articular los centros educativos con otras organizaciones, para generar espacios de vinculación dentro de las mismas escuelas (Heras Monner Sans y Miano, 2017).

\section{RQ2. ¿Qué prácticas o factores favorecen la participación social o comunitaria?}

De acuerdo con las investigaciones revisadas para impulsar la participación social, es necesario impulsar proyectos participativos y significativos, empoderar las funciones y el entendimiento de la democraciay la participación, promover actitudes de liderazgo en el personal directivo, generar espacios para que las personas agentes de la educación sean escuchadas y tomadas en cuenta, reconocer las características del contexto de los centros educativos, así como generar prácticas que reconozcan y resignifiquen la organización comunitaria (Garcés Meneses, 2017; Gómez y Bermúdez Urbina, 2015; Hernández, 2013; Perales Mejía, 2014; Pérez Galván y Ochoa Cervantes, 2017).

Al revisar las practicas exitosas de participación social, se encontró que estas buscan nuevas formas o modelos de participación que involucren a toda la comunidad educativa. Se identifica a las comunidades de aprendizaje como un modelo de participación que fortalece las relaciones entre las personas que integran una comunidad educativa.

\section{RQ3. ¿Qué prácticas o factores limitan la participación social o comunitaria?}

Se detallan como principales obstáculos para la participación los siguientes (Arzola Franco, 2014; Barrientos et al., 2016; Pérez Galván y Ochoa Cervantes, 2017; Ramírez-González, 2015; Sánchez Oñate et al., 2016; Valdés Cuervo y Sánchez, 2016):

- Homogenización de las prácticas participativas (Arzola Franco, 2014).

- La estructura jerárquica de las instituciones educativas (Arzola Franco, 2014; Pérez Galván y Ochoa Cervantes, 2017).

- Visiones y creencias tradicionales o limitadas acerca del papel de la familia en la escuela y sobre la participación, que se reflejan en actitudes de desinterés por parte de las familias o de resistencia por parte del personal de las escuelas (Barrientos et al., 2016; Pérez Galván y Ochoa Cervantes, 2017; Sánchez Oñate et al., 2016; Valdés Cuervo y Sánchez, 2016).

- Falta de comunicación entre escuela y comunidad que se refleja en la omisión o incumplimiento de las directrices (Ramirez-González, 2015).

- Relaciones debilitadas entre familia y escuela (Ramírez-González, 2015). 
http://doi.org/10.15359/ree.24-3.16

http://www.una.ac.cr/educare

educare@una.ac.cr

- Estilo directivo de carácter informativo o carente de liderazgo (Barrientos et al., 2016; Sánchez Oñate et al., 2016; Valdés Cuervo y Sánchez, 2016).

- Gestión débil del centro educativo, reflejada en una falta de cohesión (RamírezGonzález, 2015; Sánchez Oñate et al., 2016).

RQ4, ¿Cuáles son los principales retos que afrontan las instituciones educativas en cuanto a la participación social o comunitaria?

La participación de la comunidad escolar plantea muchos desafíos en la actualidad. Como refieren las investigaciones realizadas, existen múltiples obstáculos y barreras para la vinculación de la escuela y la comunidad. Uno de los mayores desafíos es impulsar proyectos de participación que redunden en experiencias significativas que contrarresten las prácticas y esquemas tradicionales de participación (Pérez Galván y Ochoa Cervantes, 2017). Para ello es necesario concientizar a padres y madres de familia y docentes para derribar las creencias y resistencias que limitan el desarrollo de prácticas participativas (Hernández, 2013; Martin y Guzmán Flores, 2016). En el ámbito administrativo, el mayor reto es generar una estructura que impulse la participación y favorezca la diversidad de prácticas y la adaptación de estas mismas al contexto escolar (Arzola Franco, 2014; Garcés Meneses 2017).

\section{Conclusiones}

La participación social ha significado, en los últimos años, un tema de interés para la comunidad científica internacional; no obstante, aún presenta áreas de oportunidad para realizar futuras investigaciones. Es importante señalar que México se destaca como uno de los países con mayor número de exploraciones en relación con la problemática; sin embargo, sus estudios se han centrado en conocer la situación de la participación social en la actualidad. En cambio, es posible encontrar propuestas para impulsar la participación social o comunitaria en países con un menor número de investigaciones y experiencias en este sentido. Otro dato relevante es que a pesar de existir un número significativo de investigaciones sobre la participación social o comunitaria en Iberoamérica, la cantidad de estudios que se realizan en el ámbito educativo a nivel básico es considerablemente menor.

En conjunto, los resultados de la revisión sistemática de bibliografía apuntan al reconocimiento de múltiples barreras para la vinculación entre escuela y comunidad, como resultado de las visiones y prácticas tradicionales en torno al papel de las personas agentes de la educación y las formas de participación de la familia y la comunidad dentro de la escuela. No obstante, es posible identificar, a partir de las propuestas existentes, una serie de factores que promueven actitudes participativas en los centros educativos. Por ello el tema de la participación social mantiene vigencia dentro de la investigación en el ámbito educativo. 
http://doi.org/10.15359/ree.24-3.16

\section{Discusión}

En un contexto cambiante en términos sociales, económicos y culturales, la escuela se ha convertido en un espacio de oportunidad para formar a estudiantes que puedan enfrentarse a las demandas del entorno que les rodea. Esto implica que la escuela se apropie de la democracia deliberativa, impulsando procesos de toma de decisiones que incluyan a toda la comunidad educativa y abriendo canales de comunicación entre todos sus integrantes, a fin de debatir, discutir o consensuar las políticas del establecimiento educativo (Garcés Meneses, 2017). En este sentido, la participación social adquiere relevancia al permitir generar espacios en los que las personas agentes de la educación puedan expresarse y ser escuchadas. En suma, se ha constatado que la participación es un elemento importante para el desempeño académico del alumnado (Valdés Cuervo y Sánchez, 2016), puesto que esto se relaciona con las altas expectativas que los padres y madres desarrollan en relación con el nivel educativo que sus hijos e hijas alcanzarán.

Impulsar la participación social implica generar distintas experiencias que permitan reconstituir el espacio educativo, a fin de dar respuesta a las necesidades de la escuela y la comunidad. Por ello es importante trascender las prácticas de gestión institucionalizadas por la política educativa (Hernández, 2013). La participación social supone un elemento de cambio dentro del sistema educativo, al tiempo que se apoya en la continuidad de la incorporación de las familias en la vida escolar. Aunque desde la perspectiva burocrática ha derivado en un compromiso más bien teórico, al tratarse de modelos que no siempre pueden aplicarse en la vida cotidiana de los centros educativos (Perales Mejía y Escobedo Carrillo, 2016). Al perder de vista el contexto que rodea a la población escolar, la política de participación social pierde relevancia ante las personas encargadas de llevarla a cabo, al tiempo que su potencial transformador se diluye hasta convertirse en un mero trámite.

Impulsar la participación de las comunidades escolares se ha convertido en todo un reto, ya que no basta con transformar la legislación educativa, sino que también hay que impulsar cambios en las creencias de las personas agentes de la educación. Actualmente, se ha desarrollado un acuerdo implícito en el que docentes, padres y madres de familia se han distanciado, lo que permite al equipo de maestros y maestras mantener el control de los asuntos escolares y a los padres y madres de familia no asumir responsabilidades adicionales a las que implica el sostener a su familia y evitar relacionarse con agentes servidores que, desde su perspectiva, no les hacen caso (Martin y Guzmán Flores, 2016). Entonces, la visión que se tiene del papel de las familias en la educación continúa siendo tradicionalista, ya que no se las considera como posibles agentes de cambio, ni se valora su papel en la transformación y mejora de la escuela, o la experiencia que pueden aportar a la mejora de las prácticas de enseñanza y los proceso de gestión escolar (Valdés-Cuervo y Sánchez, 2016). 
http://doi.org/10.15359/ree.24-3.16

http://www.una.ac.cr/educare

educare@una.ac.cr

Desde esta perspectiva, construir espacios de participación implica voltear la mirada hacia los padres y madres, para reconocerles como pares en el proceso educativo, comprender que desde su mirada y experiencia pueden enriquecer los procesos de análisis y reflexión de los procesos educativos, no solo en términos de gestión, sino también contribuir y apoyar en la práctica educativa.

Es importante identificar, entonces, que la participación social es un proceso complejo, donde las relaciones entre las mujeres y hombres agentes de la educación están marcadas por matices distintos derivados de las expectativas, creencias, experiencias y formas de comunicarse. Más allá de los cambios técnicos y modificaciones legislativas, organizar y motivar la participación social requiere de reconocer la diversidad de los contextos en los que se lleva a cabo (Perales Mejía, 2014). Por ello, los programas o proyectos orientados a vincular la escuela y la comunidad deben ser flexibles y acoplados a las necesidades y características específicas de cada caso.

En consecuencia, los proyectos participativos apelan a una gestión democrática, que involucre a todos los sujetos que conforman la escuela y reconoce el protagonismo del estudiantado, permitiéndole vivir experiencias de formación ciudadana de una manera significativa y activa (Pérez Galván y Ochoa Cervantes, 2017). Particularmente, en educación básica es importante promover la participación del alumnado en este tipo de proyectos, ya que este grupo constituye el centro de las actividades pedagógicas y es su principal beneficiario.

En suma, las investigaciones abordadas en esta revisión bibliográfica demuestran que aún existe una importante área de oportunidad en el desarrollo de proyectos participativos o propuestas para impulsar la generación de espacios de participación social y comunitaria. Por ello, se sugiere en futuras investigaciones abordar esta clase de propuestas o generar estudios desde el enfoque metodológico de investigación-acción, a fin de generar alternativas para impulsar la participación social y estrategias educativas innovadoras (Ramírez-Ramírez y Ramírez-Montoya, 2018).

\section{Declaración de Material complementario}

Este artículo tiene disponible como material complementario:

- La versión preprint del artículo en https://doi.org/10.5281/zenodo.3366344

\section{Referencias}

Aguilera Eguía, R. (2014). ¿Revisión sistemática, revisión narrativa o metaanálisis? Revista de la Sociedad Española del Dolor, 21(6), 359-360. https://www.resed.es/revista 20142168

Arzola Franco, D. M. (2014). La distancia entre el discurso de la participación y las prácticas participativas en los centros de educación secundaria. Revista Mexicana de Investigación Educativa RMIE, 19(61), 511-535. http://www.redalyc.org/articulo.oa?id=14030110008 
http://doi.org/10.15359/ree.24-3.16 http://www.una.ac.cr/educare educare@una.ac.cr

Barrientos Piñeiro, C., Silva García, P. y Antúnez Marcos, S. (2016). El papel directivo y la promoción de la participación de las familias y la comunidad en las escuelas básica. El caso de la comuna de Panguipulli-Chile. REICE. Revista Iberoamericana Sobre Calidad, Eficacia y Cambio en Educación, 14(3), 145-165. https://doi.org/10.15366/reice2016.14.3.008

Beltrán, Ó. A. (2005). Revisiones sistemáticas de la literatura. Revista Colombiana de Gastroenterología, 20(1), 60-69. http://www.scielo.org.co/pdf/rcg/v20n1/v20n1a09.pdf

Estrada Ruiz, M. J. y Sánchez Parra, Ó. A. (2016). Representaciones sobre la participación social en la educación en consejos escolares en el norte de México. Foro de Educación, 14(20), 383-403. https://doi.org/10.14516/fde.2016.014.020.019

Garcés Meneses, J. A. (2017). El reto de las escuelas democráticas modernas. El caso colombiano. Diálogos sobre Educación, 8(15), 1-15. https://doi.org/10.32870/dse.v0i15.567

Gómez, J. B. y Bermúdez Urbina, F. M. (2015). La participación de padres de familia en la educación indígena intercultural bilingüe (EIB) en la región Ch'ol de Chiapas. Revista Interamericana de Educación de Adultos, 37(1), 20-40. http://www.redalyc.org/articulo.oa?id=457544923003

Heras Monner Sans, A. I. y Miano, A. (2017). Educación, autoorganización y territorio. Revista Mexicana de Investigación Educativa, 22(73), 533-564. https://www.comie.org.mx/revista/ v2018/rmie/index.php/nrmie/article/view/24/24

Hernández, C. A. (2013). Modalidades de participación social en educación básica. Argumentos, 26(72), 167-189. $\quad$ http://www.scielo.org.mx/scielo.php?script=sci arttext\&pid $=$ S0187-57952013000200009

Latapí Sarre, P. (2005). La participación de la sociedad en la definición de políticas públicas de educación: El caso de Observatorio Ciudadano de la Educación. Perfiles Educativos, 27(107), 7-39. http://www.scielo.org.mx/pdf/peredu/v27n107/n107a02.pdf

López-Márquez, N. G. (2017). Funcionalidad familiar y participación escolar de las familias de niños con discapacidad. IE Revista de Investigación Educativa de la REDIECH, 8(14), 111-128. http://www.redalyc.org/articulo.oa?id=521653267018

Martin, C. J. y Guzmán Flores, E. (2016). La participación de madres y padres de familia en la escuela: Un divorcio de mutuo consentimiento. Sinéctica, Revista Electrónica de Educación, 46, 1-23. http://www.redalyc.org/pdf/998/99843455004.pdf

Martín, Á. y Morales, J. A. (2013). La apertura de los centros educativos a su entorno: Los centros de educación secundaria. Perspectiva Educacional, Formación de Profesores, 52 (1), 68-96. https://www.redalyc.org/pdf/3333/333328169004.pdf

Organisation for EconomicCo-operacition and Development. (2015). Education policy outlook 2015: Making reforms happens. Autor. 
http://doi.org/10.15359/ree.24-3.16

http://www.una.ac.cr/educare

educare@una.ac.cr

Payà Rico, A. y Tormo Tormo, M. (2016). La participación educativa de las familias en una escuela pública valenciana. Un estudio cualitativo. Foro de Educación, 14(21), 227-248. https://doi. org/10.14516/fde.2016.014.021.012

Perales Mejía, F. de J. (2014). La participación social en educación: Entre el habitus comunitario y la obligación administrativa. CPU-e, Revista de Investigación Educativa, 19, 86-119. https:// doi.org/10.25009/cpue.v0i19.967

Perales Mejía, F. de J. y Escobedo Carrillo, M. M. (2016). La participación social en la educación: Entre propuestas innovadoras y tradición educativa. Revista Electrónica de Investigación Educativa, 18(1), 69-81. http://www.scielo.org.mx/pdf/redie/v18n1/v18n1a5.pdf

Pérez Galván, L. M. y Ochoa Cervantes, A. de la C. (2017). La participación de los estudiantes en una escuela secundaria: Retos y posibilidades para la formación ciudadana. Revista Mexicana de Investigación Educativa, 22(72), 179-207. http://www.scielo.org.mx/pdf/rmie/ v22n72/1405-6666-rmie-22-72-00179.pdf

Pinto Contreras, R. (2013). Construyendo currículum emergente en Llaguepulli. Revista de Estudios y Experiencias en Educación, 12(24), 15-36. http://www.rexe.cl/ojournal/index. php/rexe/article/view/65/61

Ramírez-González, A. (2015). Evaluación diagnóstica de la relación escuela y comunidad: El caso de una escuela en Santo Domingo del Roble de Santa Bárbara de Heredia. Revista Electrónica Educare, 19(1), 43-65. https://doi.org/10.15359/ree.19-1.3

Ramírez-Ramírez, L. N.y Ramírez Montoya, M. S. (2018). El papel de las estrategias innovadoras en educación superior: Retos en las sociedades del conocimiento. Revista Pedagogía, 39(104), 147-170. http://saber.ucv.ve/ojs/index.php/rev ped/article/view/15707/144814482360

Sánchez Oñate, A., Reyes Reyes, F. y Villarroel Henríquez, V. (2016). Participación y expectativas de los padres sobre la educación de sus hijos en una escuela pública. Estudios Pedagógicos, 42(3), 347-367. https://doi.org/10.4067/S0718-07052016000400019

Secretaría de Educación Pública. (2016). Indicadores del ciclo funcional de los consejos escolares de participación social en la educación. informe del ciclo Escolar 2014-2015. Autor.https:// www.consejosescolares.sep.gob.mx/work/models/conapase/Resource/525/2/images/ Indicadores\%20del\%20Ciclo\%20Funcional\%20de\%20los\%20Consejos\%20Escolares\%20 2014-2015.pdf

Sierra, R. (2012). La sociedad global y el alcance de las estructuras normativas. Eidos, 17, 224255. https://dialnet.unirioja.es/ejemplar/343334

Valdés Cuervo, Á. A. y Sánchez, P. A. (2016).Las creencias de los docentes acerca de la participación familiar en la educación. REDIE. Revista Electrónica de Investigación Educativa, 18(2), 105115. http://www.redalyc.org/pdf/155/15545663008.pdf 